\title{
SUR L'APPLICATION DU PRINCIPE DE MINIMUM À LA THÉORIE DES OSCILLATIONS PROPRES DES SYSTĖMES.
}

\author{
Par \\ NICOLAS KRYLOFF \\ à KIEFF (Ucraine). \\ Extrait d'une lettre adressée à M. Nörlund.
}

Très honoré Collègue,

Dans le mémoire: „Utber die Anwendung der Variationsrechnung in der Theorie der Eigenschwingungen und über neue Klassen von Funktionalgleichungen», paru récemment ${ }^{1}$ dans les "Acta mathematica" M. le Prof. R. Courant a établi certains théorèmes d'existence, d'une grande généralité, se rapportant à la théorie des oscillations propres.

La méthode utilisée par l'éminent géomètre de Göttingen est basée essentiellement sur l'emploi du théorème concernant la possibilité d'extrạire des suites uniformément convergentes d'une suite de fonctions également continues et uniformement bornées. Ce théorème d'Arzela est d'une grande utilité pour la démonstration des théorèmes d'existence, mais pour l'appréciation de l'erreur commise en s'arrêtant à la $n^{\text {me }}$ approximation une autre voie serait, ce me semble, préférable et je me permets dans cette lettre de vous communiquer l'évaluation de l'erreur susdite en s'arrêtant aux exemples typiques, traités par M. Courant lui-même dans le mémoire ci-dessus mentionné.

Le problème peut être posé de la manière que voici: l'existence des valeurs singulières et des fonctions singulières étant supposée établie, on se propose de les calculer à l'aide du procédé des différences, utilisé par M. Courant, avec une précision voulue; ceci prouvera entre autre la convergence du procédé; en outre,

1 t. 49 , p. I-68. 
la démonstration ci-dessous développée, à de légers changements de détail près, se prête ${ }^{1}$ aussi, remarquons le en passant, à l'établissement des théorèmes d'existence dont le mémoire de $M$. Courant a été l'objet. Dans un travail ${ }^{2}$, qui sera bientôt publié et quj est écrit en collaboration avec mon élève M. Nicolas Bogoliouboff nous avons élaboré, par les mêmes procédés, pour la méthode des différences finies et ses différentes généralisations, les appréciations de l'erreur commise à la $n^{\text {me }}$ approximation dans le calcul des valeurs singulières du paramètre, des fonctions singulières et de l'intégrale de l'équation non homogène, et ceci, dans le cas, bien entendu, différent de celui de la résonnance.

Dans son mémoire $\mathbf{M}$. Courant établit ${ }^{3}$ l'existence des valeurs singulières (Eigenwerte) du paramètre $\lambda_{i}$, et des fonctions singulières (Eigenfunktion) $\varphi_{i}(x)$, relatives au système différentiel

$$
\left\{\begin{array}{l}
\left(p u^{\prime}\right)^{\prime}=-\lambda \cdot u+q u \\
p(\mathrm{o}) u^{\prime}(\mathrm{o})=t u(\mathrm{o}) ; p(\mathrm{I}) u^{\prime}(\mathrm{I})=-t^{\prime} u(\mathrm{I})
\end{array}\right.
$$

en partant du problème de minimum lié, posé pour l'intégrale

$$
D[\varphi]=\int_{0}^{1}\left(p \varphi^{\prime 2}+q \varphi^{2}\right) d x+t \varphi(0)^{2}+t^{\prime} \varphi(\mathrm{I})^{2}
$$

respectivement sous les conditions

$$
H[\varphi]=\int_{0}^{1} r \varphi^{2} d x=\mathrm{I} ; H\left[\varphi, \varphi_{i}\right]=\int_{0}^{1} r \varphi \varphi_{i} d x=0 ; i=1,2,3, \ldots, k-1
$$

Ce résultat étant acquis on peut élaborer un procédé de démonstration (valable aussi pour la démonstration des théorèmes d'existence, comme nous l'avons déjà dit précédemment) pour apprécier la différence la suite).

${ }^{1}$ il suffit de considérer $D_{n+m}[y]$ et $D_{n}[y]$ au lieu de $D[y]$ et $D_{n}[y]$ (v. les notations dans

${ }^{2}$ Ainsi ont été examinées à ce point de vue la méthode ordinaire des différences finies (principe de Lord Rayleigh), la méthode des tronçons, qui contient la méthode précédente comme eas particulier, et enfin une méthode, que nous avons nommée celle des differences supérieures, et qui, tout en présentant pour le wboundary value problem» une certaine analogie avec celle de M. Störmer, permet, dans l'appréciation de l'erreur, d'élever l'ordre de l'approximation.

${ }^{8}$ v. p. p. $49-56$ de son mémoire. 
Sur l'applicat. du princ. de minim. à la théorie des oscill. propres des systèmes. 137

$$
\lambda_{k}-\lambda_{k}^{(n)}
$$

de la $k^{\text {me }}$ valeur singulière et de sa $n^{\text {me }}$ approximation; pour cette dernì̀re on prendra ici, à l'exemple de M. Courant, la valeur de minimum lié, posé pour la somme

$$
D_{n}[\varphi, \varphi]=\Delta \sum_{v=0}^{n-1} p_{v}\left[\frac{\varphi_{v+1}-\varphi_{v}}{\Delta}\right]^{2}+\Delta \sum_{v=1}^{n-1} q_{v} \varphi_{v}^{2}+t \varphi_{0}^{2}+t^{\prime} \varphi_{n}^{2}
$$

sous les conditions:

(5) $\quad \sum_{\nu=0}^{n-1} r_{v} \varphi_{v}^{2} \Delta=H_{n}[\varphi]=\mathrm{I} ; \sum_{v=1}^{n-1} r_{v} \varphi_{v} \varphi_{v, i} \Lambda=H_{n}\left(\varphi, \varphi_{i}\right)=0 ; i=\mathrm{I}, 2,3, \ldots, k-\mathrm{I}$

ce qui aura lieu pour les valeurs $\varphi_{v}, \nu=\mathrm{I}, 2, \ldots, n$, que nous prendrons pour les sommets d'une ligne polygonale, dénotée par $\varphi_{k}^{(n)}(x)$ dans la suite.

Cela étant considérons deux fonctions $F_{1}(x)$ et $F_{2}(x)$ ainsi formées:

$$
\begin{aligned}
& F_{1}(x)=\sum_{i=1}^{k} A_{i} \varphi_{i}(x) \\
& F_{2}(x)=\sum_{i=1}^{k} B_{i} \varphi_{i}^{(n)}(x)
\end{aligned}
$$

et choisissons les constantes $A_{i}, B_{i}$ de sorte que:

$$
\begin{aligned}
H_{n}\left[F_{1}\right]=\sum_{\nu=1}^{n-1} r_{\nu} F_{1, \nu}^{2} A=\mathrm{I} ; H_{n}\left[F_{1}, \varphi_{i}^{(n)}\right] & =\sum_{\nu=1}^{n-1} v_{\nu} F_{1, \nu} \varphi_{\nu, i}^{(n)} \mathcal{A}=\mathrm{o} ; \\
i & =\mathrm{I}, 2,3, \ldots, k-\mathrm{I} ; F_{1, \nu}^{\prime}=F_{1}\left(x_{v}\right)
\end{aligned}
$$

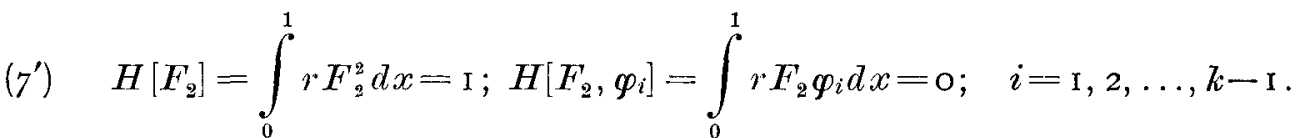

Alors en vertu même de la notion de minimum on a:

$$
\begin{aligned}
& D_{n}\left[F_{1}, F_{1}\right] \geq \lambda_{k}^{(n)} \\
& D\left[F_{2}\right] \geq \lambda_{k}
\end{aligned}
$$

ou, ce qui est identique,

18-2822. Acta mathematica. 52. Imprimó lo 23 août 1928. 


$$
\begin{aligned}
& D\left[F_{1}\right]+\left\{D_{n}\left[F_{1}, F_{1}\right]-D\left[F_{1}\right]\right\} \geq \lambda_{k}^{(n)} \\
& D_{n}\left[F_{2}, F_{2}\right]+\left\{D\left[F_{2}\right]-D_{n}\left[F_{2}, F_{2}\right]\right\} \geq \lambda_{k} .
\end{aligned}
$$

Or

$$
\begin{aligned}
& D\left[F_{1}\right]=\sum_{i=1}^{k} A_{i}^{2} \lambda_{i}<\lambda_{k} H\left[F_{1}\right]=\lambda_{k}\left\{\mathrm{I}+\left(H\left[\boldsymbol{F}_{1}\right]-H_{n}\left[\boldsymbol{F}_{1}\right]\right)\right\} \\
& D_{n}\left[\boldsymbol{F}_{2}, \boldsymbol{F}_{2}\right]=\sum_{i=1}^{k} B_{i}^{2} \lambda_{i}^{(n)}<\lambda_{k}^{(n)} H_{n}\left[\boldsymbol{F}_{2}^{\prime}\right]=\lambda_{k}^{(n)}\left\{\mathrm{I}+\left(H_{n}\left[\boldsymbol{F}_{2}\right]-H\left[F_{2}\right]\right)\right\}
\end{aligned}
$$

car il est bien évident, que

$$
\int_{0}^{1}\left[p \varphi_{i}^{\prime} \varphi_{j}^{\prime}+q \varphi_{i} \varphi_{j}\right] d x+t \varphi_{i}(\mathrm{o}) \varphi_{j}(\mathrm{o})+t^{\prime} \varphi_{i}(\mathrm{I}) \varphi_{j}(\mathrm{I})=\left\{\begin{array}{lll}
\mathrm{o}, & \text { si } & i \neq j \\
\lambda_{k}, & \text { si } & i=j
\end{array}\right.
$$

et

(9) $\quad \sum_{\nu=0}^{n-1} p_{v} \frac{\Delta \varphi_{v, i}^{(n)}}{\mathcal{J}} \cdot \frac{\Delta \varphi_{v, j}^{(n)}}{\mathcal{A}} \Delta+\sum_{v=1}^{n-1} q_{v} \varphi_{v, i}^{(n)} \varphi_{v, j}^{(n)} A+t \varphi_{0, i}^{(n)} \varphi_{0, j}^{(n)}+t^{\prime} \varphi_{n, i}^{(n)} \varphi_{n, j}^{(n)}= \begin{cases}\mathrm{o}, & \text { si } i \neq j \\ \lambda_{k}^{(n)}, & \text { si } i=j\end{cases}$

done

$$
\lambda_{k}\left\{\mathrm{I}+\left(H\left[F_{1}\right]-H_{n}\left[F_{1}\right)\right)\right\}+\left(D_{n}\left[F_{1}, F_{1}\right]-D\left[F_{1}\right]\right) \geq \lambda_{k}^{(n)} ;
$$

$$
\lambda_{k}^{(n)}\left\{\mathrm{I}+\left(H_{n}\left[\boldsymbol{F}_{2}\right]-H\left[\boldsymbol{F}_{2}\right]\right\}+\left(D\left[\boldsymbol{F}_{2}\right]-D_{n}\left[\boldsymbol{F}_{2}, \boldsymbol{F}_{2}\right]\right) \geq \lambda_{k} .\right.
$$

Or d'après le théorème des accroissements finis on a:

$$
\left|H_{n}\left[F_{1}\right]-H\left[F_{1}\right]\right| \leq \Lambda \int_{0}^{1}\left|\frac{d\left(r F_{1}^{2}\right)}{d x}\right| d x \leq \int_{0}^{1} \frac{\left|r^{\prime}\right| F_{1}^{2}+\left|2 r F_{1}\right|\left|F_{1}^{\prime}\right| d x}{n},
$$

d'autre part on a évidemment:

$$
\int_{0}^{1} \varphi_{i}^{2} d x<\frac{\mathrm{I}}{\min r}, \quad\left(\operatorname{car} \int_{0}^{1} r \varphi_{i}^{2} d x=\mathrm{I}\right) \text { et } \int_{0}^{1} p \varphi_{i}^{\prime 2} d x>(\min p) \int_{0}^{1}{\varphi^{\prime}}_{i}^{2} d x
$$

par conséquent, de la relation évidente 
Sur l'applicat. du princ. de minim. à la théorie des oscill. propres des systèmes. 139

$$
\varphi^{2}(x)=\varphi^{2}(\xi)+2 \int_{\xi}^{x} \varphi(x) \varphi^{\prime}(x) d x
$$

on tire, vu les formules (13) et (8):

$$
\left.\int_{0}^{1} \varphi_{i}^{\prime 2} d x<\frac{\lambda_{i}}{\min p}+\frac{\left[|t|+\left|t^{\prime}\right|\right]}{\min \frac{1}{p}}\left[\left(\frac{\mathrm{I}}{\min r}\right)+2\right] \sqrt{\int_{0}^{1} \varphi_{i}^{\prime 2} d x} \cdot \frac{\mathrm{I}}{\sqrt{\min r}}\right]+\frac{\max |q|}{\min r^{\prime} \cdot \min p}
$$

done

(14) $\int_{0}^{1} \varphi_{i}^{\prime 2} d x<\left[\frac{|t|+\left|t^{\prime}\right|}{\min p \cdot \sqrt{\min r}}+\right.$

$$
\left.+\sqrt{\left\{\frac{\lambda_{i}}{\min p}+\frac{|t|+\left|t^{\prime}\right|}{\min p \min r}+\frac{\max |q|}{\min r \cdot \min p}\right\}+\left\{\frac{|t|+\left|t^{\prime}\right|}{\min p \sqrt{\min r}}\right\}^{2}}\right]^{2}=C_{1}
$$

par conséquent

$$
\int_{0}^{1} F_{I}^{\prime 2} d x \leq \sum_{i=1}^{k} A_{i}^{2} \int_{0}^{1} \sum_{i=1}^{k} \varphi_{i}^{\prime 2} d x<k C_{1} H\left[F_{1}\right]
$$

car

$$
\int_{0}^{1} r \mathscr{F}_{i} \varphi_{j} d x=\left\{\begin{array}{lll}
\text { o, } & \text { si } & i \neq j \\
\text { I, } & \text { si } & i=j
\end{array}\right.
$$

donc de la formule (12) on tire au moyen de l'inégalité de Schwarz:

(I 5) $\quad\left|H_{n}\left[F_{1}^{\prime}\right]-H\left[F_{1}\right]\right|<\frac{\mathrm{I}}{n}\left[\frac{\max \left|r^{\prime}\right|}{\min |r|}+2 \max |\sqrt{r}| \sqrt{k C_{1}}\right] H\left[F_{1}\right]=\frac{C_{2} H\left[F_{1}\right]}{n}$

c.-à-d., vu $\left(6^{\prime}\right)$ :

$$
\left|H\left[F_{1}\right]\right|<\frac{\mathrm{I}}{\mathrm{I}-\frac{C_{2}}{n}}
$$

done 


$$
\left|H_{n}\left[F_{1}\right]-H\left[F_{1}\right]\right|<\frac{\frac{C_{2}}{n}}{\mathbf{I}-\frac{C_{2}}{n}}=\frac{C_{2}}{n-C_{2}}
$$

et

(17)

$$
\int_{0}^{1} F_{1}^{\prime 2} d x<\frac{k C_{1}}{\mathrm{I}-\frac{C_{2}}{n}}=C_{3}
$$

D'une manière analogue on trouve:

$$
\left|H_{n}\left[F_{2}\right]-H\left[F_{2}\right]\right|<\frac{C_{4}}{n-C_{4}}, \quad \text { (où } C_{4}=\text { const.) }
$$

Cela étant, observons que

(19) $\quad\left|D_{n}\left[F_{1}, F_{1}\right]-D\left[F_{1}\right]\right| \leq \frac{1}{n}\left[\left|p^{\prime}\right| \int_{0}^{1} F_{1}^{\prime 2} d x+\right.$

$$
\left.+2|p| \int_{0}^{1}\left|F_{1}^{\prime} F_{1}^{\prime \prime}\right| d x+\int_{0}^{1}\left|q^{\prime}\right| F_{1}^{2} d x+\int_{0}^{1} 2\left|q F_{1} F_{1}^{\prime}\right| d x\right]
$$

d'autre part

$$
\int_{0}^{1} F^{\prime \prime 2} d x \leq \sum_{i=1}^{k} A_{i}^{2} \sum_{i=1}^{k} \int_{0}^{1} \varphi_{i}^{\prime \prime 2} d x<H\left[F_{1}\right] k \cdot\left[\max \int_{0}^{1} \varphi_{i}^{\prime \prime 2} d x\right] ; \quad i=\mathrm{I}, 2, \ldots, k ;
$$

or d'après l'équation différentielle vérifiée par $\varphi_{k}$ et d'après l'inégalité (I4) on a:

$\left(19^{\prime}\right)$

$$
\int_{0}^{1} \varphi_{i}^{\prime \prime 2} d x<2\left\{\max \left[\frac{\lambda_{i} r^{r}+q}{p \sqrt{r}}\right]^{2}+\max \left|\frac{p^{\prime}}{p}\right|^{2} C_{1}\right\}=C_{5}\left(\lambda_{i}\right),
$$

donc de l'inégalité (I9) on tire, vu les inégalités ( $\left.14^{\prime}\right),\left(15^{\prime}\right),\left(19^{\prime}\right)$ :

(20) $\quad\left|D_{n}\left[F_{1}, F_{1}\right]-D\left[F_{1}\right]\right|<\frac{\mathrm{I}}{n}\left[\max \left|p^{\prime}\right| . k C_{1}+\right.$

$$
\left.+2 \max \left|\frac{q}{\sqrt{r}}\right| \sqrt{k C_{1}}+\max \left|\frac{q}{r}\right|+2 \max |p| \sqrt{k C_{1}} \cdot \sqrt{k C_{5}\left(\lambda_{k}\right)}\right] H\left[F_{1}\right]<\frac{C_{6}}{n}
$$


Sur l'applicat. du princ. de minim. à la théorie des oscill. propres des systèmes. 141

où

$$
C_{6}=\max \left|p^{\prime}\right| . k C_{1}+2\left[\max \left|\frac{q}{\sqrt{r}}\right|+\max |p| k C_{5}\left(\lambda_{5}\right) \sqrt{k C_{1}}+\max \left|\frac{q}{r}\right|\right] \frac{\mathrm{I}}{\mathrm{I}-\frac{C_{2}}{n}} .
$$

D'une manière analogue on trouve:

$$
\left|D\left[F_{2}\right]-D_{n}\left[F_{2}, F_{2}\right]\right|<\frac{C_{7}}{n}, \quad \text { où } C_{7}=\text { const. }
$$

Ceci étant établi, on tire des inégalités (Io) et (I I), vu (I6), (20), (I8), (2I):

$$
\left|\lambda_{k}-\lambda_{k}^{(n)}\right|<\lambda_{k}^{(n)}\left[\frac{C_{2}}{n-C_{2}}+\frac{C_{4}}{n-C_{4}}\right]+\frac{C_{6}}{n}+\frac{C_{7}}{n}
$$

Cette formule (22) fournit une limitation de l'erreur obtenue à la $n^{\text {me }}$ approximation pour la différence

$$
\left|\lambda_{k}-\lambda_{k}^{(n)}\right|
$$

sous forme explicite, où figurent les constantes $C_{2}, C_{4}, C_{6}, C_{7}$, qu'on détermine d'après les formules précédemment établies en vertu des données du problème. Il va sans dire, qu'il est aisé d'obtenir, dans la formule (22) les limitations plus serrées, si l'on fait sur les fonctions $p(x), q(x), r(x)$ les hypothèses correspondantes.

La démonstration précédente, bien entendu, peut être érigée en celle de l'existence des valeurs et des fonctions singulières, comme on l'a déjà précédemment remarqué; mais ceci est superflu, étant donné que la démonstration basée sur l'emploi du théorème d'Arzela, comme par ex. celle de $\mathbf{M}$. Courant, est plus courte. La démonstration de la formule (22) peut être aisement généralisée pour traiter des cas plus généraux, comme par ex. "die allgemeineren Probleme» de M. Courant ${ }^{1}$, mais les formules ainsi obtenues sont par trop longues pour trouver place dans cette lettre.

Kieff, le I octobre I927.

1 V. Ibidem p. p. $56-60$. 